\title{
Split-screen single-camera stereoscopic PIV application to a turbulent confined swirling layer with free surface
}

\author{
Philippe M. Bardet • Per F. Peterson • \\ Ömer Savaş
}

Received: 5 March 2009/Revised: 22 December 2009/Accepted: 11 January 2010/Published online: 29 January 2010

(C) The Author(s) 2010. This article is published with open access at Springerlink.com

\begin{abstract}
An annular liquid wall jet, or vortex tube, generated by helical injection inside a tube is studied experimentally as a possible means of fusion reactor shielding. The hollow confined vortex/swirling layer exhibits simultaneously all the complexities of swirling turbulence, free surface, droplet formation, bubble entrapment; all posing challenging diagnostic issues. The construction of flow apparatus and the choice of working liquid and seeding particles facilitate unimpeded optical access to the flow field. A split-screen, single-camera stereoscopic particle image velocimetry (SPIV) scheme is employed for flow field characterization. Image calibration and free surface identification issues are discussed. The interference in measurements of laser beam reflection at the interface are identified and discussed. Selected velocity measurements and turbulence statistics are presented at $\operatorname{Re}_{\lambda}=70$ ( $\operatorname{Re}=3500$ based on mean layer thickness).
\end{abstract}

\section{Introduction}

In various proposed inertial fusion energy schemes, fusion reactions result from compression of fuel pellets by magnetic fields, lasers, or heavy-ion beams. Surfaces facing the

P. M. Bardet $(\square)$

California Institute of Technology,

Graduate Aerospace Laboratories, Pasadena, CA, USA

e-mail: p_bardet@yahoo.com

P. F. Peterson

Department of Nuclear Engineering, University of California,

Berkeley, CA, USA

Ö. Savaş

Department of Mechanical Engineering,

University of California, Berkeley, CA, USA reactions are exposed to high energy neutrons, alpha particles, gamma-rays, X-rays, and ions following deuteriumtritium nuclei fusion. To avoid costly development of materials that can withstand these conditions, interpositioning of thick liquid-salt flow to shield chamber structures are considered. This scheme is commonly referred to as thick liquid protection (Moir et al. 1994; Peterson 2001). To protect focusing magnets in certain heavy-ion fusion chamber designs, inner surface of heavy-ion beam tube is covered with a swirling, turbulent, annular wall jet, called a vortex tube (Pemberton et al. 2003; Yu et al. 2003). To maximize neutron shielding, the liquid layer must be positioned close to the heavy-ion beam and must have a smooth free surface. Due to the high Prandtl number of proposed liquid-salts $(\sim 20)$, however, a high free surface renewal rate is desirable to condensate vaporized coolant that can enter the beam port. Current configuration is numerically studied by Gulec et al. (2000) using re-normalization group (RNG) k- $\varepsilon$ turbulence model. Turbulence data are not available. It is, however, well known that the concave geometry of the flow geometry has a destabilizing effect on the fluid layer, therefore each specific flow case must be carefully studied. It is unlikely that general turbulence models are applicable (Hoffman et al. 1985).

Flow characteristics of a 1:4 scale model of a prototype nozzle designed to generate a vortex tube is studied experimentally, (Fig. 1a). Liquid is injected through a regular array of holes tangent to the inner tube surface (Fig. 1b) and at a $45^{\circ}$ angle from the pipe axis (Fig. 1c), resulting in a unity-nominal swirl ratio, defined as $S=\left\langle U_{\theta}\right\rangle_{r} /\left\langle U_{z}\right\rangle_{r}$. The array of holes is made of twelve rows and nine columns. The holes in the first four and last four columns point in opposite axial direction, while the holes in the center column alternativerly point in opposite axial directions, (Fig. 1a, c, and d). The nozzle generates two 
(a)

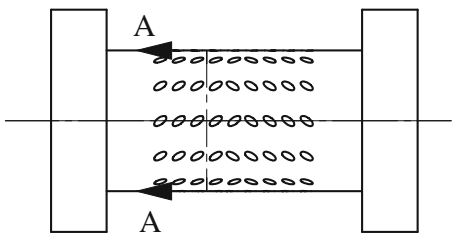

(b) Section A-A

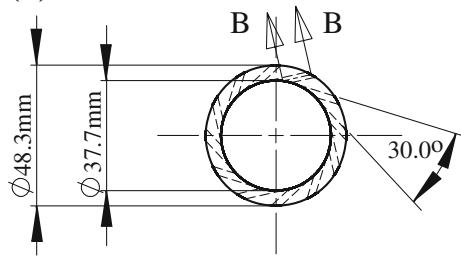

(c) Section B-B

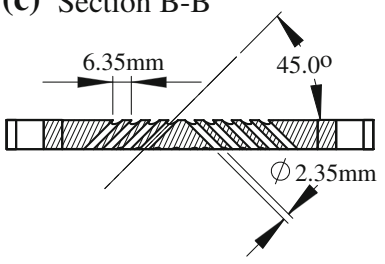

(d)

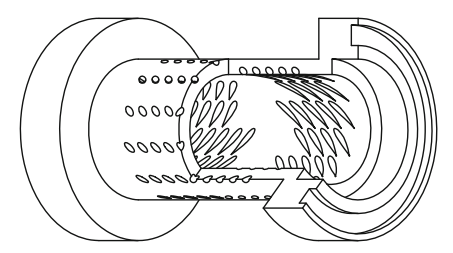

(e)

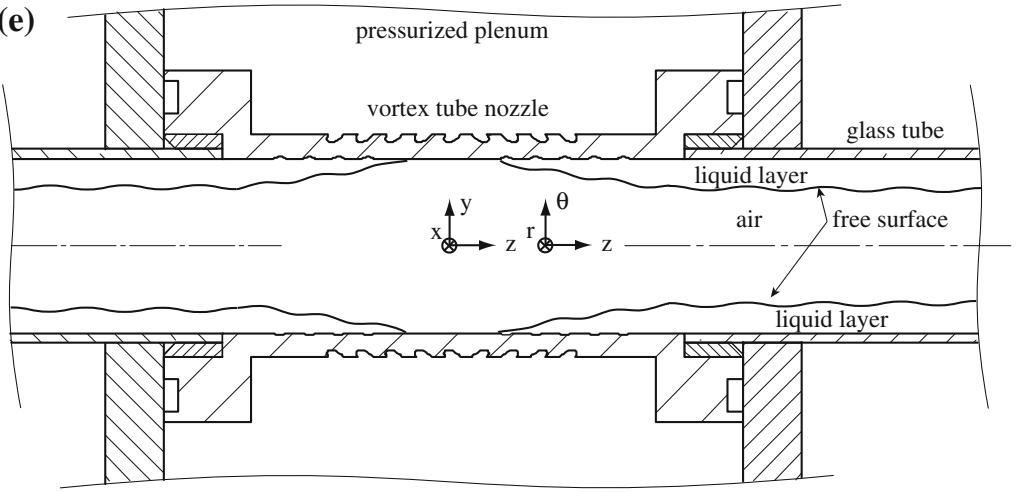

Fig. 1 Vortex tube nozzle geometry. a Top view, b cross-section A-A, $\mathbf{c}$ cross-section B-B, $\mathbf{d}$ perspective view with a section of the nozzle cut away to reveal the nozzle inner surface, $\mathbf{e}$ cross-section of the assembled nozzle and tubes, with reference axis

antisymmetric annular liquid layers. A 38-mm inner diameter glass tube is connected at both extremities of the nozzles (Fig. 1e), the resulting liquid layers are about $7.3 \mathrm{~mm}$ thick. A low viscosity, high index of refraction mineral oil was used to simulate high-temperature liquidsalt. To take advantage of the optical properties of the oil, a fused silica test section with a refractive index similar to the oil was employed. Figure 2 shows sample average and instantaneous shapes of the free surface as photographed through the layer.

To study the highly three dimensional and turbulent flow field, a single-camera split-screen stereoscopic particle image velocimetry (SPIV) system based on angular displacement was developed. Single camera SPIV allows saving money on hardware by only employing one camera. That technique has been employed previously for lateral displacement SPIV by Arroyo and Greated (1991) and for angular displacement by Grant et al. (1991). Special calibration targets were manufactured for in situ camera calibration. Fluorescent seeds in conjunction with sharp cutoff filters were employed to block glare reflected from the free surface. Flow field measurement in the vicinity of the free surface were successfully obtained in the presence of strong surface irregularities. The geometry of the flow imposed certain constraints that limit the measurement accuracy. The focus of the paper is on the instrumentation and measurement techniques. Only sample measurements and turbulence statistics obtained from SPIV measurements are presented.
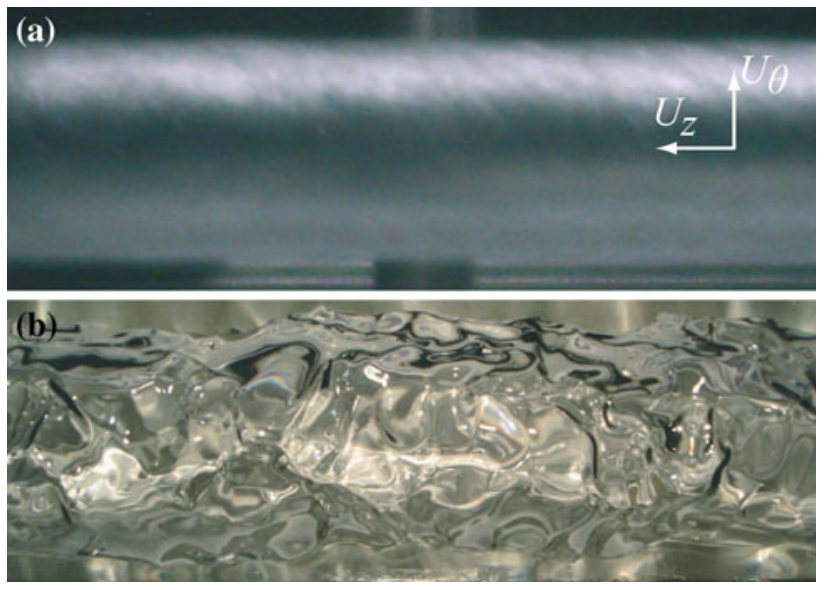

Fig. 2 Vortex tube free surface pictures at 10 tube diameters from inlet. a Time-averaged free surface as perceived by the naked eyes (30-ms exposure time). b Instantaneous surface frozen by a $\sim 5 \mu \mathrm{s}$ camera flash light

\section{Working fluid}

The working fluid for the experiment is chosen to meet various thermal and optical requirements. The fluid property requirements for flow and thermal similarity are discussed elsewhere (Bardet and Peterson 2008). The primary optical requirement is to match the index of refraction of the test section material for undistorted optical access to the flow. The test section wall material and fluid refractive indices are matched by employing fused silica tube and windows ( $n=1.4575$ at $489 \mathrm{~nm}$ ), and a light mineral oil, 
Penreco $^{\circledR}$ Drakesol $^{\circledR}$ 260AT $(n=1.4498$ at $489 \mathrm{~nm}$ and $20^{\circ} \mathrm{C}$ ), similar to the fluid used in the Idaho National Laboratory Matched Index of Refraction (MIR) facility (Stoots et al. 2001).

This oil is stable, inexpensive, and safe to handle, in fact it is the main component of baby oils. It can be considered Newtonian and its low viscosity (Table 1) makes it a good liquid candidate for turbulence experiments, while its low surface tension can be beneficial for free surface experiments. Since the viscosity of the oil changes significantly with temperature (Table 1), the temperature and the flow injection rate are controlled individually to independently vary the injection Reynolds number $R_{i n j}$ and turbulence Reynolds number $\operatorname{Re}_{\lambda}$. A costly unfavorable aspect of the oil is that it bleaches the fluorescent particles used for PIV if they are left in solution for more than 4 weeks. This bleaching, however, occurs in a matter of minutes at temperatures higher than $70^{\circ} \mathrm{C}$. High-quality plastics, such as viton, must be employed with this oil or they will either extend or harden. Drakesol ${ }^{\circledR} 260$ AT is manufactured by oil cracking, and as a consequence its chemical composition and thermophysical properties vary slightly from one batch to another. This requires that its thermophysical properties must be measured for the particular batch used in an experiment, as was done here.

\section{SPIV}

\subsection{SPIV review}

Particle image velocimetry (Adrian 1991, 2005; Cho 1989) has become the de facto $2 \mathrm{D}$ velocity field measurement technique in fluid mechanics. Judicious exploitation of PIV data contamination by out-plane velocity has led to SPIV, which extracts the complete velocity vector on a plane. SPIV can be performed with two optical configurations: lateral and angular displacements (Prasad 2000). In the lateral displacement_or lens translation—technique object, lens, and image planes are parallel and laterally shifted with respect to one another. As a result, the cameras do not need calibration, but the maximum viewing angles are limited. In the angular displacement technique, there is no limitation on the viewing

Table 1 Penreco ${ }^{\circledR}$ Drakesol ${ }^{\circledR}$ 260AT thermophysical properties relevant for this study, as measured by the authors. $\rho, v$, and $\sigma$ are the oil density, kinematic viscosity, and surface tension respectively

\begin{tabular}{llll}
\hline$T\left({ }^{\circ} \mathrm{C}\right)$ & $\rho\left(\mathrm{kg} \mathrm{m}^{-3}\right)$ & $v\left(10^{6} \mathrm{~m}^{2} \mathrm{~s}^{-1}\right)$ & $\sigma\left(\mathrm{N} \mathrm{m}^{-1}\right)$ \\
\hline 25 & 792.5 & 5.4 & 0.027 \\
40 & 785 & 3.9 & 0.026 \\
60 & 775 & 2.7 & 0.024 \\
\hline
\end{tabular}

angles, which can be set at their optimum value of $45^{\circ}$, but the cameras need calibration. The object plane can be made in focus without large depth of field (obtained by small aperture lens) if the Scheimpflug condition is implemented. Angular displacement SPIV has been used with (Willert 1997) and without (Lawson and Wu 1997; Westerweel and Nieuwstadt 1991) satisfying this condition.

\subsection{Angular displacement single camera SPIV}

When feasible, it is beneficial to project both views onto a single camera sensor in SPIV. In particular, if the measurement field has a high aspect ratio, it is simply an efficient use of the camera sensor area. Lateral displacement SPIV technique has been implemented with a single camera (Arroyo and Greated 1991) in which a set of four mirrors are used to project both views onto the camera sensor plane. This configuration is, however, somewhat intricate to implement because it requires the installation of two mirrors between the lens and the sensor chip. Further, it suffers from the limitations associated with lens translation.

Figure 3 shows the angular displacement SPIV configuration employed in this study. Two first surface mirrors and a right angle prism mirror are used to project both views onto a single camera. The viewing angles $\alpha_{1}$ and $\alpha_{2}$ are nominally $45^{\circ}$. The optical path is equivalent to having two virtual cameras recording the flow. This split-screen imaging technique was first employed for SPIV using a 35-mm film camera by Grant et al. (1991). The film was then digitized for processing. This configuration is inexpensive, easy to install, and align. However, it requires a longer optical path than if using 2 cameras and therefore a longer focal length lens. Scheimpflug condition could be satisfied but it would require the use of 4 prisms with two positioned between the lens and the camera sensor, rendering it rather impractical. This configuration was also employed for stereogrammetry methods (Grant et al. 1995) for flow visualization experiments with reflective flakes (Jacob and Savaş 1995) and for 3D flow measurement (Racca and Dewey 1988; Peskin 1972).

\subsection{Free surface handling}

The laser sheet is set to coincide with the tube axis to perform measurements in the radial plane, hence, normal to the mean free surface seen in Fig. 2a. The instantaneous surface shape undulations seen in Fig. 2b, necessitate particular precautions to prevent unavoidable reflections from interfering with measurements, even damaging optical components and the camera sensor.

The approach employed here is to separate the illumination and observation wavelengths by employing fluorescent particles and sharp cutoff filters. The illuminating 

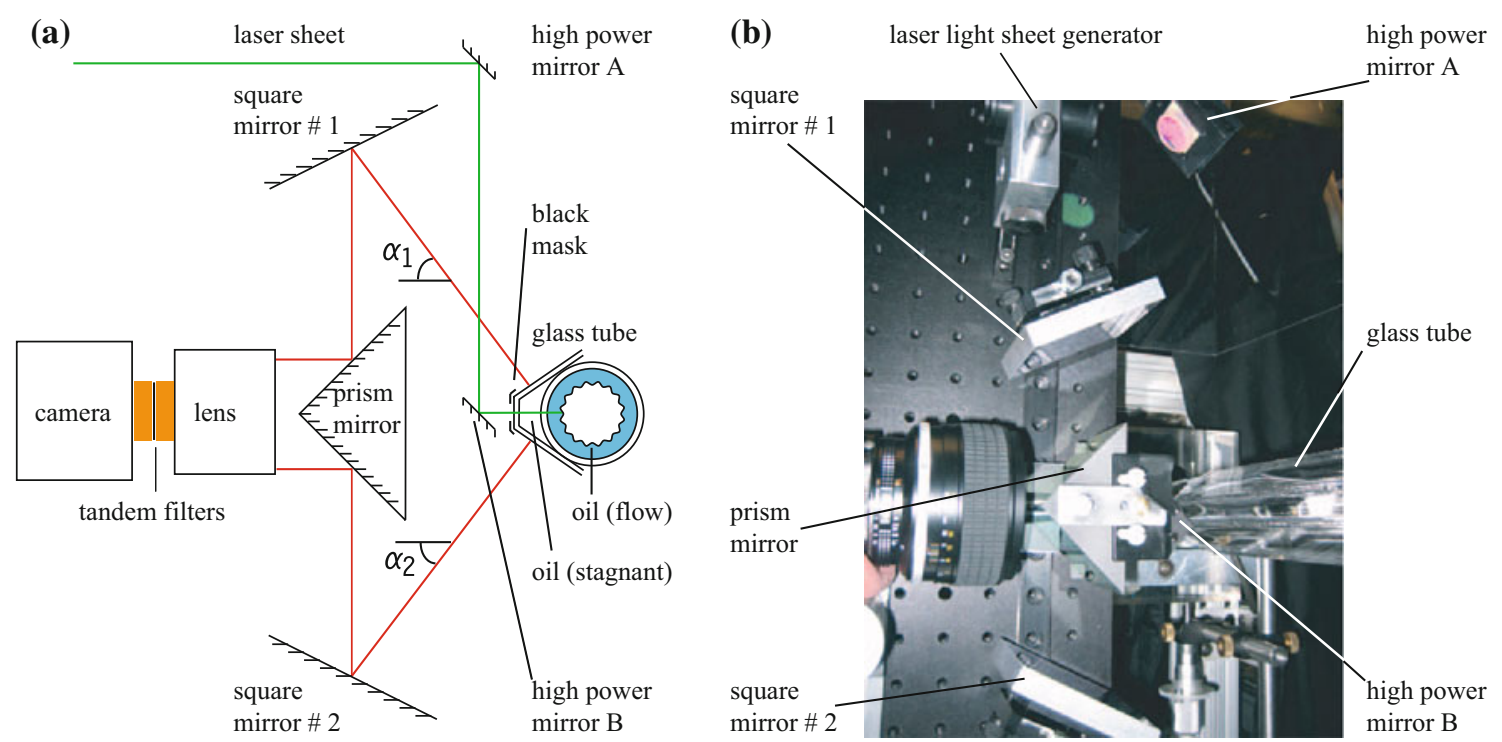

Fig. 3 a Split-Screen Single Camera Stereoscopic PIV optical configuration. b Photograph of the setup
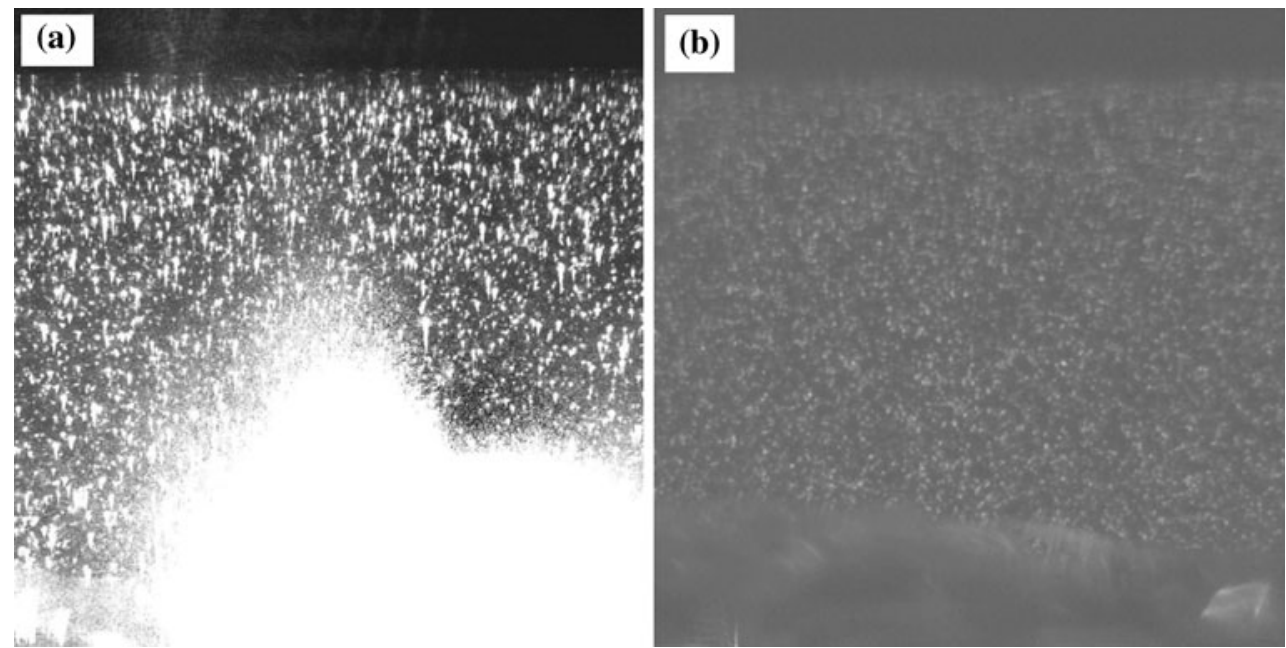

Fig. 4 PIV images (a) without optical cutoff filter: glare is visible at the bottom of the frame, (b) with optical filters: glare is suppressed

laser is a double pulsed Nd-Yag laser of $532 \mathrm{~nm}$ wavelength (New Wave, Gemini-30). The working fluid is seeded with dry polymer fluorescent particles of $7 \mu \mathrm{m}$ mean diameter and $1.05 \mathrm{~g} / \mathrm{cm}^{3}$ density (Duke Scientific 36-2, proprietary dye). The Stokes shift, or the difference between the maxima of absorption and emission spectra, is $70 \mathrm{~nm}$, i.e. the seeds emit $602 \mathrm{~nm}$ red/orange light. A longpass filter with a cutoff wavelength of $570 \mathrm{~nm}$ (Schott OG570) is used to isolate the fluoresced light from the laser light.

The cutoff filters are made of a glass matrix embedded with light-absorbing semiconductor colloidal particles, which implies that the absorbed light intensity increases with the filter thickness (Bindra et al. 1999; Oak et al. 1993). To take advantage of this property, two 3-mm thick filters are used in series between the lens and the camera CCD sensor (Fig. 3a). With this optical arrangement, no direct laser light interfered with flow field images (Fig. 4).

\section{Displacements reconstruction}

In extracting the three components of displacement vectors, image planes must be corrected for nonuniform magnification, which is done by calibrating the camera and warping the images. 


\subsection{Calibration}

The mapping between the image and object planes can be determined by geometrical reconstruction, $2 \mathrm{D}$ or $3 \mathrm{D}$ calibration (Prasad 2000). Due to spatial constraints imposed by the apparatus, the $2 \mathrm{D}$ backprojection calibration method is used here.

The calibration target is a custom manufactured $1.5-\mathrm{mm}$ thick and 25.4-mm square fused silica plate with an index of refraction nearly matching that of the working fluid. On one side of the plate a square grid of $508 \mu \mathrm{m} \pm 2.5 \mu \mathrm{m}$ pitch is laser etched by a laser scribing company-mrlaser.com-(Fig. 5a). The width of the grid lines, essentially shallow channels, are measured with a microscope to be $178 \mu \mathrm{m}$. The target is first covered with a $\sim 150$-nm thick layer of gold-palladium by ion sputtering. Then, the plate surface is wiped clean to leave the gold-palladium layer in the channels only, resulting in a target pattern that looks like a dark square mesh on a transparent background (Fig. 5b). To identify corners of the square grid, each view of the calibration target is enhanced by contrast gamma stretching followed by a median filter to remove salt and pepper noise; the views are then cross-correlated with a corner mask, the correlation peaks indicating the corner locations. Subpixel calibration accuracy is obtainable by curve fitting of the local maxima, however this is not implemented here.

\subsection{Warping}

Of the two common techniques of 3D displacement construction, mapping of the velocity vectors after SPIV processing or warping the SPIV images before processing (Prasad 2000), the latter was implemented here despite being computationally intensive, since it allows for correcting for misalignments that might result from the calibration step (Willert 1997).

A polynomial mapping function is commonly used for backprojecting the particle images (Prasad 2000). A pair of bilinear equations are employed here

$x=a_{0}+a_{1} X+a_{2} Y+a_{3} X Y$

$y=b_{0}+b_{1} X+b_{2} Y+b_{3} X Y$

where $(x, y)$ is a point on the object plane, and $(X, Y)$ on the image plane (Gonzales and Woods 2001). The coefficients $a_{i}$ and $b_{i}$ are estimated through a linear least-mean-square regression analysis using more than 40 points per view of the calibration images. The particles images are then reconstructed using a cubic convolution interpolation technique. Bilinear warping maps nonplanar quadrilaterals (Wolberg 1990). Bilinear mapping preserves straight lines that are vertical and horizontal. Like affine transformations, points along those lines that are equispaced in the source image remain equispaced in the mapped image. Lines that are diagonal, however, are mapped into quadratic curves. Dewarped pictures of the calibration target, with view \#1 flipped horizontally, are shown in Fig. 5c. On both views, similar points overlap well within one pixel. Higher-order polynomials were tried, with less satisfactory results. The three displacement components are then reconstructed following Willert's geometry (Willert 1997).

\subsection{PIV processing}

The flow has simultaneous features of being turbulent, having a solid wall, and possessing a very irregular free surface. Thus, the interrogation of the particles images calls for particular considerations. To accommodate strong gradients that are expected in turbulent flows, special PIV processing algorithms have been developed. They include, but are not (a)

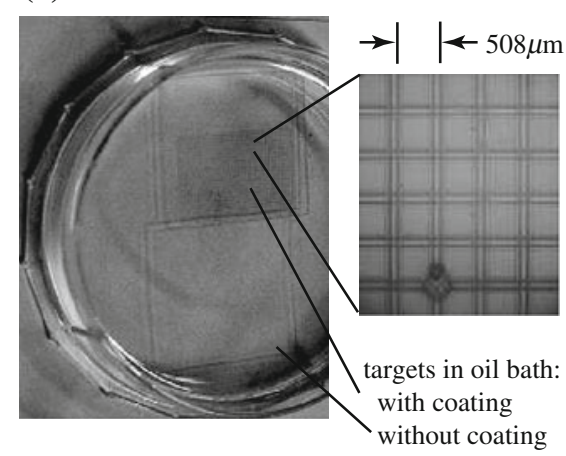

(b)

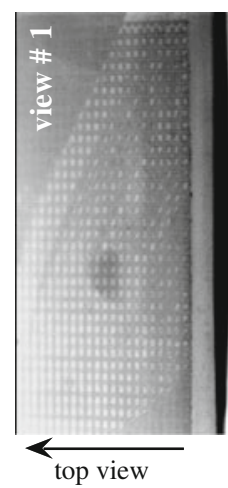

Fig. 5 Image calibration. a Custom made coated and uncoated calibration targets, in oil bath and detail. b Calibration images. The left view corresponds to the top view or image \#1 in Fig. 3a. c Dewarped images, gird corners overlap within a pixel. View \# 1 is

(c)
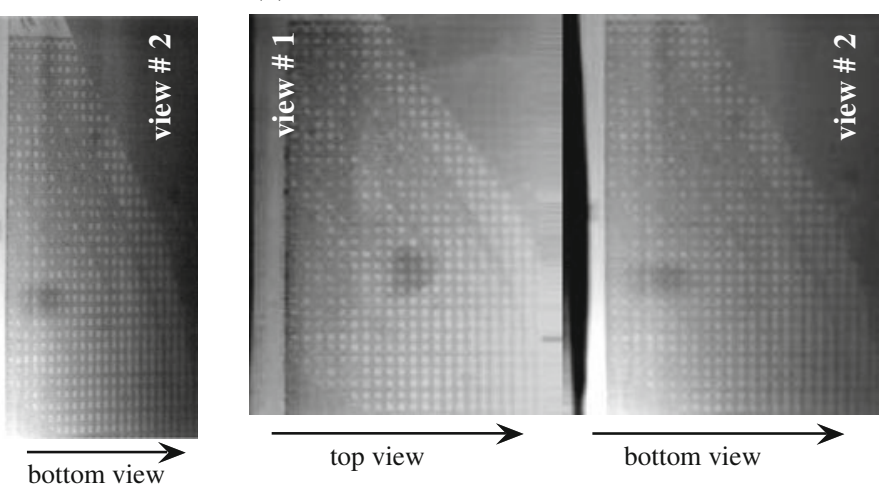

flipped horizontally. The misaligned diffuse dark patches in the images (b) and (c) are likely due to a suspended piece of debris in the fluid along the optical path 
limited to, particle image deformation (Huang et al. 1993a, b), super-resolution PIV (Keane et al. 1995), direct estimation of velocity gradients (Mayer 2002), and adaptive Lagrangian parcel tracking (aLPT) by Sholl and Savaş (1997).

aLPT algorithm is employed here. This algorithm utilizes interrogation windows that are advected and deformed according to the local velocity and velocitygradient fields, improving the quality of the data in regions of strong deformation. The outputs of the algorithm are the two-dimensional velocity vector field and its gradient tensor (which is computed spectrally). The vorticity is determined from the velocity-gradient tensor. The algorithm allows for dynamic resizing of interrogation window. Cross-correlations, derivatives, and filtering are computed with fast Fourier transforms (FFT). For this purpose, the velocity array is centered in the smallest $2^{n} \times 2^{m}$ array so that FFT algorithms can be employed for fast calculation. The data are smoothly extended to 'zero' values at the edges of the newly constructed FFT domain to insure continuity in the slopes to prevent ringing in the frequency domain. Once in the wave number domain, the transform is multiplied with the appropriate powers of the wave numbers to construct the transforms of the derivatives, which are in turn obtained by subsequent inverse transforms. Derivatives up to $3 \mathrm{rd}$ order are obtained for aLPT where they are used to reconstruct deformed interrogation windows. In particular, second order deformations are used where deformation of a rectangular fluid parcel is traced to second order which results in a cushion parcel with curved edges rather than a parallelepiped with straight parallel edges. During these calculations, the raw data are filtered in the frequency domain using a low-pass filter kernel $1-\exp \left(-1 / k^{2}\right)$ where $k$ is the modulus of the wave vector (normalized with the desired cut off wave number). Before the aLPT processing begins in earnest, a routine PIV correlation technique is used to identify outliers, which are subsequently recalculated at doubled and, if needed, quadrupled the windows. If this process also fails, the outliers are replaced with the mean of their neighboring vectors. The final output of aLPT, however is not scanned for individual outliers, but passed through a general filter as describe above. Because of its accuracy in measuring flow fields in region of strong deformation, aLPT is well suited to work on turbulent flows in general, and hence is appropriate to analyze the data of this experiment.

Before processing the data, particles images are preprocessed to increase the cross-correlation coefficients average value calculated in the PIV analysis software. Since the PIV laser employs two separate laser heads, one frame is usually slightly brighter than the other. The intensity of both frames is then corrected such that their average intensities are equal (Ortega 2001).

\section{SPIV results}

\subsection{Operational details}

In the optical setup of Fig. 3, the actual viewing angles are determined from the calibration images, and are $43.0^{\circ}$ and $41.8^{\circ}$ for views 1 and 2 , respectively. The square mirrors are set at an angle of $54^{\circ} \pm 0.5^{\circ}$ from the laser sheet. The nominal magnification is estimated from the calibration images as 0.52 .

A double exposure CCD camera (Kodak ES1.0) is employed, which has a resolution of $1008 \mathrm{~W} \times 1018 \mathrm{H}$ pixels and a depth of 8-bits. The PIV image pair acquisition rate is $2 \times 15 \mathrm{~Hz}$. A Nikkor $85 \mathrm{~mm}$ lens with extension tubes constitute the recording optics. The tandem long-pass filters are positioned inside the extension tubes. The $f$-stop (aperture setting) is set to $f / 4$ to obtain an acceptable depth of field. The recorded fields of view are about 11.5 $\mathrm{W} \times 17.5 \mathrm{H} \mathrm{mm}^{2}$ for both views.

The double-pulsed PIV laser can deliver an energy of $100 \mathrm{~mJ}$ per pulse. For this experiment, nearly $95 \%$ of this energy is used. The light sheet thickness is about $200 \mu \mathrm{m}$. The pulse separation is set to $25 \mu$ s for the data presented here. With an out-of-plane average velocity on the order of $2.5 \mathrm{~m} / \mathrm{s}$, this guarantees that the loss of pair is on the order of $30 \%$.

The image acquisition system captures 512 image-pairs for each run at a $15 \mathrm{~Hz}$ rate. Two runs are done for each flow regime. Due to the low sampling frequency of the acquisition system, the vector fields obtained are considered to be independent realizations, and hence a total of 1024 independent flow samples are acquired. The images are recorded once the flow has been established, typically a few seconds after the flow setting is dialed in.

Before processing, individual PIV views are extracted from the images and subsequently warped to correct for distortion. The final PIV images are of size $550 \mathrm{~W} \times 950$ $\mathrm{H}$ pixels. Registration shift that results from a misalignment between the laser sheet and the calibration target can be corrected by analyzing the raw SPIV images. Seeds that are bonded together create unusual bright pixels that can be identified on both views. Averaging the difference of the pixels location on each views yields the registration shift within experimental uncertainties (Fig. 6). The present analysis assumes that the laser sheet and the calibration target planes are parallel, but could be extended to the more general case where they are not parallel. In the present study, an average of 200 such pixel patches were systematically identified to correct for registration shift.

The PIV processing algorithm dynamically adjusts the interrogation window size at each PIV passes if correlation can be found. The windows are hence set between $64 \times 64$ pixels and $8 \times 8$ pixels from an initial value of 


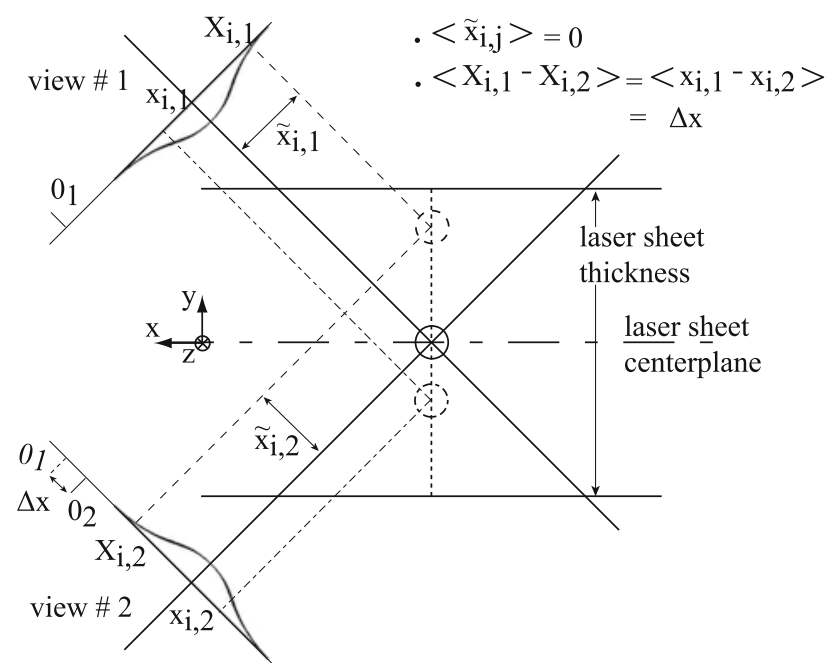

Fig. 6 Identical seeds that are not on the centerplane of the laser sheet appear at different relative position on each camera views. One can take advantage of this fact to correct SPIV data for registration shift and estimate the laser sheet thickness

$32 \times 32$ pixels. In this test case, $90 \%$ of the final windows are $16 \times 16$ pixels and $32 \times 16$ pixels. The windows steps are kept fixed at 8 pixels in both directions, which is about $140 \mu \mathrm{m}$. Finally, the vector fields are spectrally filtered with a long-pass filter to remove outliers. The PIV processing algorithm is described in great details in Sholl and Savaş (1997).

\subsection{Geometry limitations}

The combination of sharp cutoff filters and fluorescent particles proved effective in blocking glare and reflections from the free surface, Fig. 4. However, the systematic identification of this interface based on SPIV images only is rather challenging due to stereoscopic vision, finite thickness of the laser sheet, and reflected light from the free surface. In particular, the reflections can lead to spurious measurements.

The identification and reconstruction of an edge in stereoscopic imaging is a complex problem. Identifying a physical point on two images can be ambiguous and at times impossible. For example, a convex edge can be optically occlusive for one view and not the other (Faugeras 1993). Interface location recorded in either view will be displaced with respect to each other by an amount depending on the incidence angle and proportional to the laser sheet thickness.

At the interface between two media of different refractive indices, light is either reflected or transmitted or both. Snell's equations give the reflection and refraction angles, while Fresnel's laws give intensity of s- and p-polarized components of the reflected and transmitted beams (Hecht
1998). The reflectance and transmittance for the oil-air interface are shown in Fig. 7. For incident angles smaller than $40^{\circ}$, most of the light is transmitted through the interface and very little is reflected. For the most unfavorable case at the Brewster or polarization angle, $\theta_{p}=34.6^{\circ}$, the interface does not reflect any $\mathrm{p}$-polarized light, and the s-polarized reflected light intensity is less than $13 \%$. For incident angles larger than the critical angle, $\theta_{c}=43.6^{\circ}$, there is total internal reflection.

Since the viewing angles in Fig. $3\left(\alpha_{1}=43.0^{\circ}\right.$ and $\left.\alpha_{1}=41.8^{\circ}\right)$ are close to the Brewster angle $\left(\theta_{p}=34.6^{\circ}\right)$, the interface does not act as a mirror for the light scattered by the particles and regions outside the layer appear dark (Lin and Perlin 1998). In this case, the incident angle, $\theta_{i}$, employed in Fig. 7 is the angle between the normal to the free surface and the viewing angle. However, the strong free surface irregularities (see Fig. 2b), in particular troughs, locally increase the viewing angle with respect to the interface and hence act as mirrors for the fluorescent light (Fig. 8a). That is the source of the ligaments like patterns observed on the outside of the layer of view \# 2 in Fig. 9.

When the free surface forms a small angle with the incident laser sheet (laser sheet nearly normal to the interface), most of the laser light is transmitted and very little is reflected (Fig. 7, here the incident angle is taken between the incident laser sheet and the free surface normal). This is the most favorable case to conduct PIV measurements in a plane perpendicular to the average free surface location. As the degree of waviness increases, the optical geometry rapidly shifts away from this condition. In certain cases, the angle formed between the incident laser light and the free surface is such that locally total internal reflection occurs making the interface virtually disappear (Fig. 8b). This particular case renders the identification of the free surface position on the PIV images difficult. In addition for higher injection flow rates than the one treated

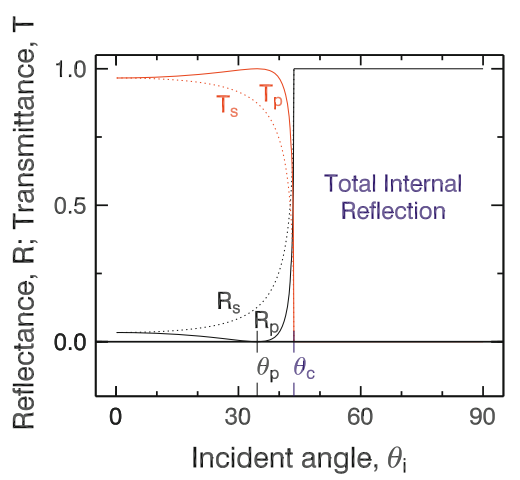

Fig. 7 Transmittance and reflectance at the mineral oil-air interface. Brewster angle $\theta_{p}=34.6^{\circ}$, and total internal reflection angle $\theta_{c}=43.6^{\circ}$ 
Fig. 8 Fluorescent and laser beam behavior at the interface. Circles represent seeds inside the investigation plane, while crosses, seeds outside this plane (a) Fluorescent light:

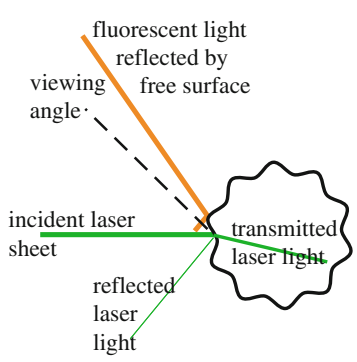

(b) Laser light with total internal reflection:

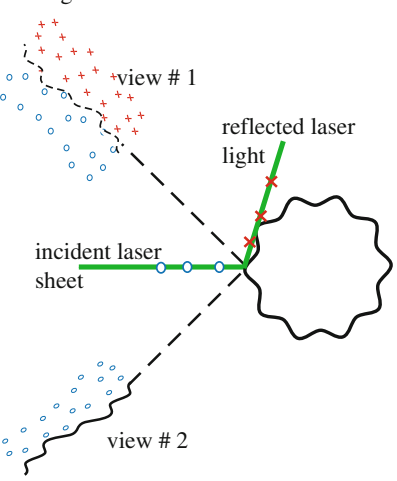

(c) Fluorescent light with total internal reflection

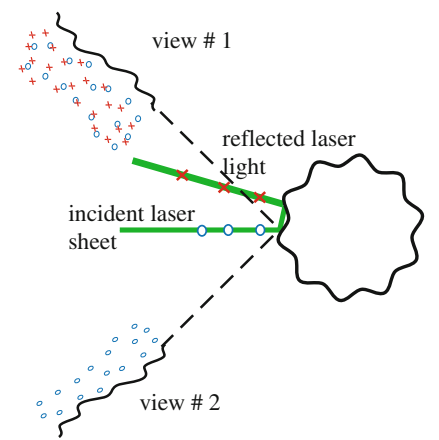

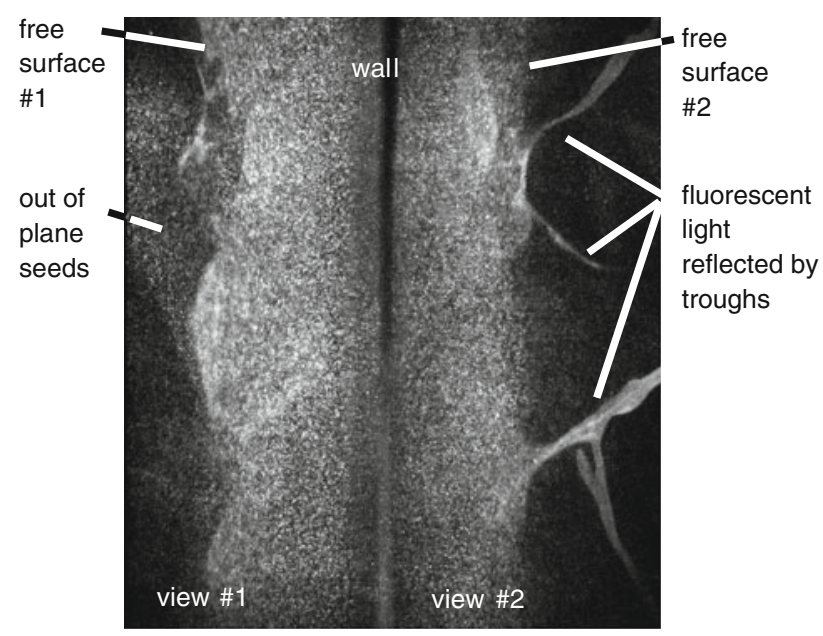

Fig. 9 SPIV particle image illustrating free surface details. Flow is from bottom to top

in this paper, drops formation and bubble entrapment contribute to blurring the interface. As a result, an edge finder algorithm that systematically tracts the liquid-air interface has not been successfully developed. A sample picture showing the out of plane particles that blur the interface position are presented in Fig. 9.

Total internal reflection at the interface also has a negative effect on flow field measurements. As illustrated in Fig. 8c, the laser sheet can be redirected inside the layer in a plane, nearly parallel to the incident sheet that can appear in focus on the object plane. Hence two planes are, at times, simultaneously recorded and manifest themselves in two ways on the data. Most frequently, this results in a local increase of background intensity and a decrease of the signal to noise ratio. However, in rare events when the liquid surface has both large axial curvature and small radial curvature, discernable out of plane seeds appear on the PIV data and lead to erroneous data. This last phenomenon could be tentatively tracked by monitoring the seed density of the PIV images and discarding SPIV samples with a statistically abnormal high seeds density. The authors have not implemented this technique as this phenomenon is very localized and appears in less than 5\% of the frames. It should be also noted that these phenomena are minimized if the Scheimpflug condition is satisfied (the object and investigation planes coincide) and a fast lens is employed.

\subsection{Error analysis}

Uncertainties in turbulent flow measurements are due to systematic errors of instruments and convergence of statistics. The measurement errors in our systems are mainly due to PIV processing algorithm (aLPT), misalignment of calibration target with laser sheet, and camera calibration uncertainties.

For the error analysis and the presentation of subsequent data, it is convenient to decompose the instantaneous velocity, $\mathcal{U}_{i}$, into an ensemble averaged portion, $U_{i} \equiv$ $\left.<\mathcal{U}_{i}\right\rangle$, and a fluctuating portion, $u_{i}$, following the Reynolds decomposition, $\mathcal{U}_{i}=U_{i}+u_{i}$. For the rms values of individual fluctuation quantities, we use $u_{i}^{\prime}=\sqrt{<u_{i} u_{i}>}$ where no summation over index $i$ is implied. The ensemble averaging $<>$ is done over 1024 independent PIV samples.

Sholl and Savaş (1997) estimated the accuracy of aLPT to be $1 \%$ of the measured velocity. The misalignment of the laser sheet and the calibration target planes are calculated analytically to have a maximal $0.5 \%$ effect on the velocity measurements. Calibration errors of camera views are estimated by varying the location of the calibration reference points and shifting the views with respect to each other, both by \pm 1 pixel (i.e. the calibration and dewarping precision). The new raw images are then dewarped and interrogated to extract the three velocity components. The percent error between the modified and original data is computed for the mean and rms velocities. The errors for $\mathcal{U}_{r}, \mathcal{U}_{\theta}$, and $\mathcal{U}_{z}$ are respectively $3.0,3.0,1.5 \%$ on the mean and respectively $3.0,3.0,1.0 \%$ on the rms.

The statistics convergence is estimated following methodology for stationary processes with Gaussian distribution and $u_{i}^{\prime}$ standard deviation around the mean, Baird (1995). 
The standard deviation on the mean and on the standard deviation are respectively $u_{i}^{\prime} / \sqrt{N}$ and $u_{i}^{\prime} / \sqrt{2(N-1)}$, with $N$ the number of samples (i.e. 1024). Thus, the standard deviation on $U_{r}$ is $5 \%$, while it is only 0.3 and $0.4 \%$ for $U_{\theta}$ and $U_{z}$. The large uncertainty for $U_{r}$ is due to the fact that $\left|U_{r}\right|$ is smaller than its rms. For the rms velocity, the standard deviations are on the order of $2.5 \%$ for $u_{r}{ }^{\prime}$ and $2.0 \%$ both for $u_{\theta}{ }^{\prime}$ and $u_{z}{ }^{\prime} .95 \%$ confidence interval is twice the standard deviations and is used to compare the instrumental and statistical precisions.

Finally, the combined uncertainty of instruments and statistics is estimated as the maximum of all errors for each component of the mean and rms velocities. Calibration uncertainties limit the accuracy of $U_{\theta}$ and $U_{z}$, while $U_{r}, u_{r}{ }^{\prime}$, $u_{\theta}^{\prime}$, and $u_{z}^{\prime}$ are limited by statistics.

\subsection{Sample results}

In the data presented below, a band of four velocity vectors have been masked around the edges of the flow fields to remove spurious vectors resulting from PIV processing near the edges of the images. The usable flow field has then $39 \mathrm{~W} \times 107 \mathrm{H}$ vectors. Further, the free surface is not systematically identified for reasons explained in Sect. 5.2 as a result this interface is masked as well.

In the experiments, PIV data are recorded at five stations for three injection flow rates and at three oil temperatures. The data presented here are acquired at about 2.4 tube diameters from the injection nozzle, with $2.1 \times 10^{-3} \mathrm{~m}^{3} / \mathrm{s}$ flow rate, and $25^{\circ} \mathrm{C}$ temperature. For this flow, the layer thickness is about $7.5 \mathrm{~mm}$. The swirl ratio is about $5 / 3$. Due to the particulars of the construction of the glass viewing prism, the flow in the immediate vicinity of the tube wall is not accessible. Thus, no reference to viscous sublayer is made.

The mean flow Reynolds number based on the average speed and the layer thickness is 3500 . The (longitudinal) Taylor microscale

$\lambda=\left[u_{z}^{\prime 2} /\left\langle\left(\frac{\partial u_{z}}{\partial z}\right)^{2}\right\rangle\right]^{1 / 2}$

is about $700 \mu \mathrm{m}, 5$ times the PIV interrogation step size. The Taylor Reynolds number, $\operatorname{Re}_{\lambda}=u^{\prime} \lambda / v$, based on the Taylor microscale, and the rms velocity $u^{\prime}=\sqrt{\frac{1}{3}<u_{i} u_{i}>}$ with summation over index, is about 70 for the dataset presented here.

\subsubsection{Flow field}

Sample velocity field vector data are shown in Fig. 10, where the wall is positioned on the left of the flow field and the free surface on the right. The mean flow is essentially an axisymmetric two-dimensional one; the azimuthal and axial velocity components, $U_{\theta}$ and $U_{z}$ are dominant. The mean radial flow, $U_{r}$, is on the order of $-5 \%$ of the mean axial flow, $U_{z}$.

Figure 10a and c show instantaneous $\mathcal{U}_{i}$ and fluctuation velocity $u_{i}$ fields of a single realization. Figure $10 \mathrm{~b}$ and $\mathrm{d}$ ) shows a magnified section taken between the region $r / R$ $=[0.95-0.73]$ and $z / R=[4.46-4.75]$ of the same instantaneous and fluctuating velocity fields. On the fluctuating fields, Fig. 10c, and particularly d, highly threedimensional structures are identifiable: including a 3D (a) Instanteneous Velocity

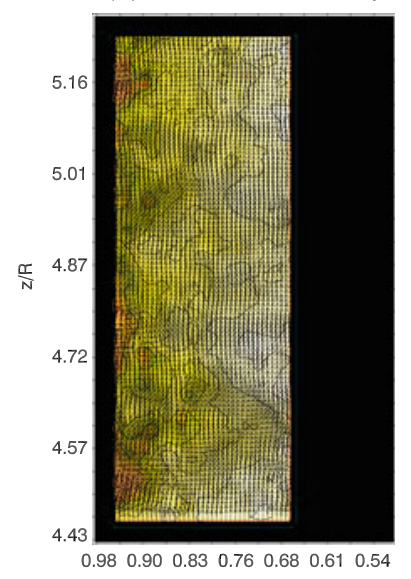

$\mathrm{r} / \mathrm{R}$ (b) Zoomed Section

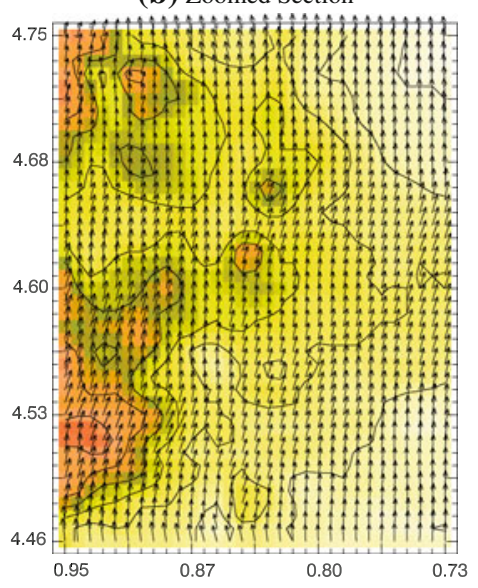

(c) Fluctuation Velocity

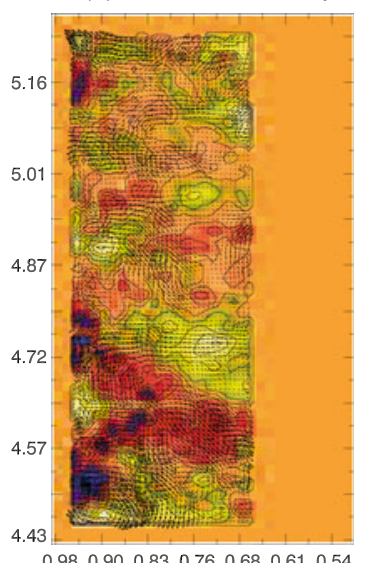

$\mathrm{r} / \mathrm{R}$ (d) Zoomed Section

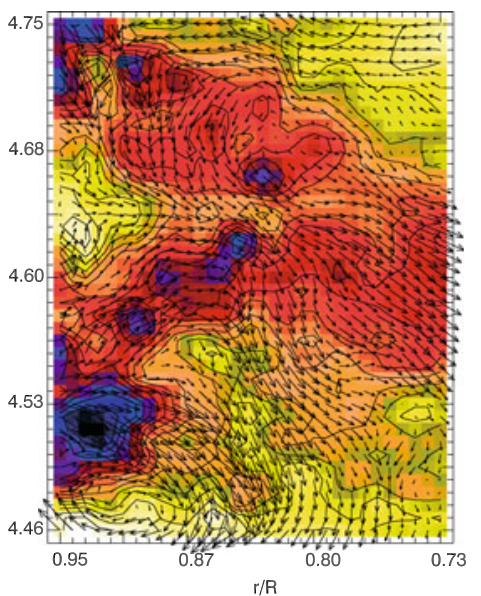

$r / R$
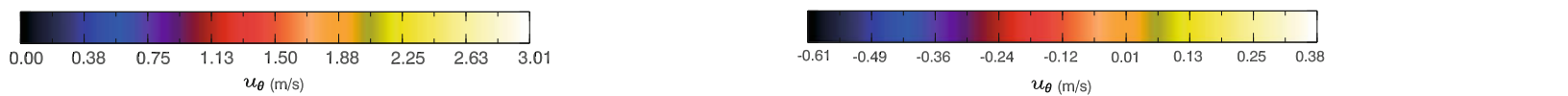

Fig. 10 Samples velocity fields. Vector plots: radial and axial components; contour plots: azimuthal component. Instantaneous velocity $\mathcal{U}_{i}:(\mathbf{a})$ whole field (b) zoomed section. Fluctuating velocity $u_{i}$ : (c) whole field (d) zoomed section 


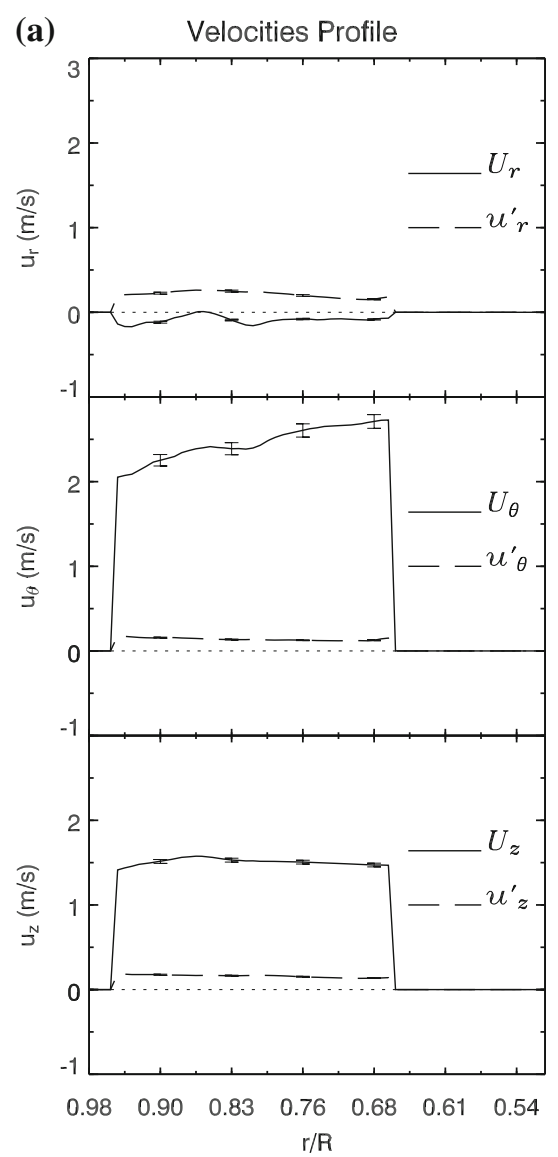

Fig. 11 Velocity field statistics. a Mean and $r m s$ velocity profiles at axial step 60 with $95 \%$ confidence interval, b instantaneous and fluctuation velocity pdf's, c fluctuation velocity pdf's nondimensionalized but their

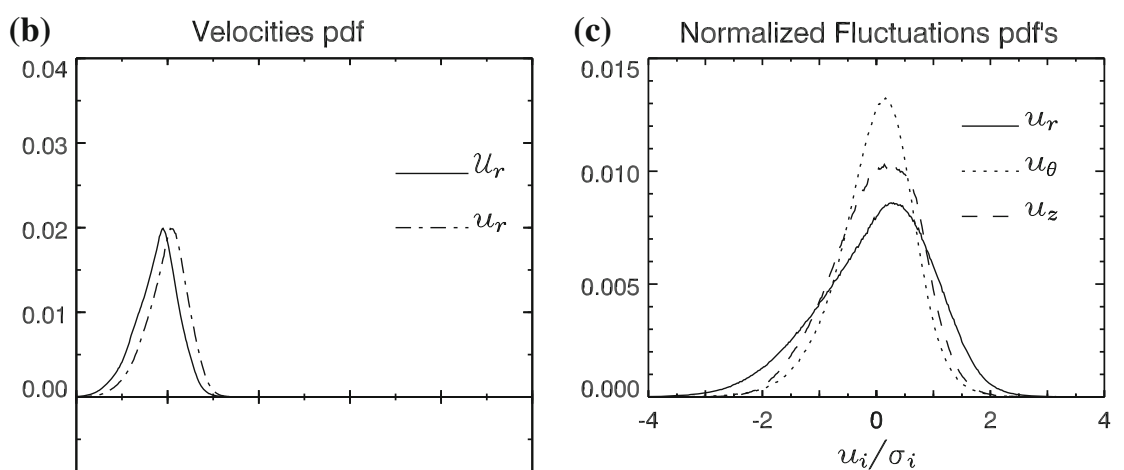

(d) 1D Wave Number Energy Spectra

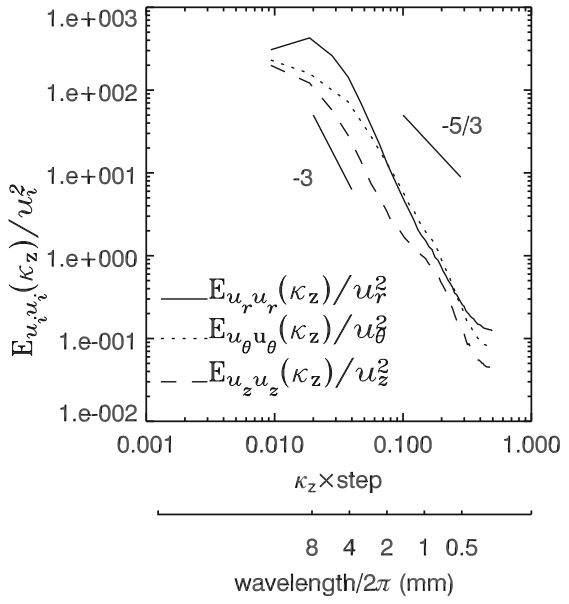

standard deviation $\sigma$, and $\mathbf{d} 1 \mathrm{D}$ wavenumber energy spectra in the axial direction, $-5 / 3$ slope is shown for reference

stagnation region at coordinates $(0.95,4.64)$ and regularly spaced cyclonic and anticyclonic circulatory flow patterns, centered at coordinates $(0.90,4.48)$ and $(0.84,4.68)$.

\subsubsection{Flow statistics}

Sample flow statistics are shown in Fig. 11. Figure 11a shows the profiles of the mean $U_{i}$ and $r m s$ velocities $u_{i}^{\prime}$ of all three components along the radial direction and at the axial step 60. The radial $r m s u_{r}^{\prime}$ is the highest of the three components. In the radial direction, the average axial velocity profile is nearly constant across the resolved flow area, while the azimuthal profile has a nearly constant gradient, with small ripples, which can be the signature of the injection jets. The small, nearly uniform, negative radial velocity profile results from shear stress at the wall and turbulent kinetic energy production, which slow down the mean flow, hence thickening the liquid layer.

Instantaneous and fluctuating velocities probability distribution functions ( $p d f^{\prime}$ 's) are displayed in Fig. 11b. The $p d f$ 's are constructed for all the points of the velocity field and from all the PIV samples, adding to a total of $39 \times$ $107 \times 1024 \sim 4.3 \times 10^{6}$ individual vectors. Figure $11 \mathrm{c}$ shows the fluctuating velocity $p d f$ 's nondimensionalized by their respective rms velocity $\left(u_{i}^{\prime}\right)$ to have the same standard deviations $\left(\sigma_{i}=u_{i}^{\prime}\right)$. All fluctuating $p d f^{\prime}$ s are positively skewed, which means that the positive velocity excursions are more likely than the negatives ones. Since $u_{r}$ has the largest standard deviation (Fig. 11a), or rms, and the largest kurtosis (i.e. 4th order moment for flatness), one expects that the contribution of the radial component to the turbulent kinetic energy is higher than other components. Further, $u_{r}$ takes larger negative than positive values.

To simplify the computation of the axial one-dimensional wavenumber energy spectra, the flow is assumed to be weakly homogeneous, i.e. the average velocities $U_{i}$ are constant and independent of location, and the covariance function is independent of location and depends only on distance between points (Shumway and Stoffer 2006). The power spectrum densities are obtained by taking the norm square of 2D fast Fourier transforms (FFT) of the velocity fields and ensemble averaging over all independent 
samples. A Hanning window is used over the edges of the velocity field. The 1D wavenumber energy spectra along the axial direction (Fig. 11d) are then extracted by taking the power spectrum densities at the zero wavenumber of the spectrum tensor, $\kappa_{r}=0$.

Even though $\mathrm{Re}_{\lambda}$ is at the threshold for the flow to be considered fully turbulent, it appears that the one-dimensional wavenumber energy spectra follow $a-3$ power law. The spectra exhibit another interesting feature. There seems to be a plateau around the wavenumber 0.1 , suggesting that a certain amount of energy is contained in this range. At the highest wavenumbers (near 0.5), the energy spectra tend toward a constant value. This behavior is unlikely to be physical and is instead an artifact of the PIV cross-correlation processing.

\subsubsection{Reynolds stress}

The Reynolds stress tensor is evaluated as

$\overline{u_{i} u_{j}}=<u_{i} u_{j}>$

Three-component, two-dimensional SPIV allows construction of the symmetric Reynolds stress tensor $\overline{u_{i} u_{j}}(r, z)$. A sample result is shown in Fig. 12. From the difference in magnitudes of the diagonal terms, it is clear that the turbulence of this swirling flow is anisotropic. $\overline{u_{i} u_{j}}$ confirms the $p d f$ 's conclusion that the radial turbulent kinetic energy has the dominant contribution. Of the off-diagonal terms, only $\overline{u_{r} u_{\theta}}$ takes significant values, indicating turbulent shear along $r, \theta$ directions. Spatial variations of Reynolds stress, which lead to turbulent transport inside the flow, appear to be consistent among all components. In particular, a local extremum is discernable at the bottom of all frames near coordinates $(0.85,4.55)$. This extremum is likely a signature of the injection jets. For this particular data set, the turbulent kinetic energy per unit mass averaged over measured field is computed to be $k$ $=0.048 \mathrm{~m}^{2} / \mathrm{s}^{2}$.

\subsection{Free surface analysis}

For reasons covered in Sect. 5.2, the free surface could not be systematically resolved from the SPIV images, instead external instantaneous pictures, such as Fig. 1b, are employed to estimate the amplitude of waves at the liquidair interface. For the particular flow conditions presented here, no drops were generated and there are no evidence of gas bubbles entrapment; the free surface irregularities variance is $4.5 \pm 1.2 \%$ of the layer thickness.

That translates to waves with $1 \mathrm{~mm}$ rms amplitude for the prototype. This small amplitude is well below the $5 \mathrm{~mm}$ standoff distance between the heavy-ion beam and the liquid vortex for the heavy-ion fusion point design

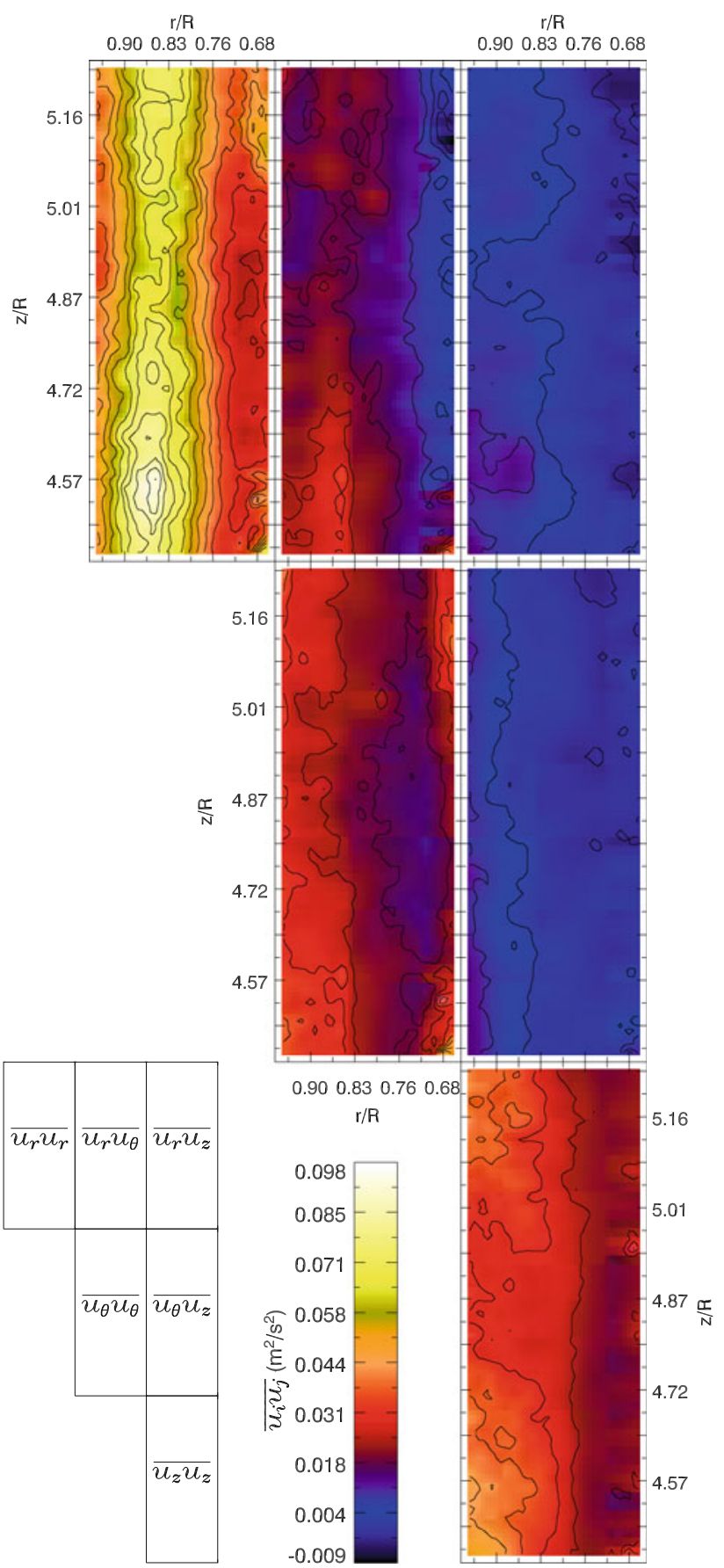

Fig. 12 Reynolds stress tensor, $\overline{u_{i} u_{j}}(r, z)$

(Yu et al. 2003) insuring that the liquid layer will not interact with the ion beam. The current design can thus be employed for neutron shielding.

\section{Conclusions}

An experimental technique is implemented for the study of a complex turbulent flow. The flow, intended for fusion 
reactor shielding applications, is essentially a confined turbulent hollow core swirling flow, or a hollow confined turbulent vortex. The coexistence of turbulence, swirl, and a free surface presents interesting fluid dynamics phenomena and challenging diagnostic problems. A single camera stereoscopic PIV technique is employed where a compact optical system facilitates radial access to the flow. An embedded calibration technique is developed. By using a combination of fluorescent particles and cut off filters, reflections form free surface are virtually prevented from illuminating the camera image sensor while successfully recording particle images for SPIV. Numerous adverse effects of light sheet reflections off the free surface are identified and discussed; the worst case being a double internal reflection projecting a sheet nearly parallel to the original incident light sheet. An adaptive PIV processing algorithm is used to explore the structure in the turbulent layer. Sample flow measurements are presented showing the nature of the flow, in particular flow statistics present evidence of anisotropy of the turbulence.

Acknowledgments We thank Dr. Carolyn White for depositing a layer of gold-palladium onto the calibration targets.

Open Access This article is distributed under the terms of the Creative Commons Attribution Noncommercial License which permits any noncommercial use, distribution, and reproduction in any medium, provided the original author(s) and source are credited.

\section{References}

Adrian RJ (1991) Particle-imaging techniques for experimental fluid mechanics. Annu Rev Fluid Mech 23:261-304

Adrian RJ (2005) Twenty years of particle image velocimetry. Exp Fluids 39:159-169

Arroyo MP, Greated CA (1991) Stereoscopic particle image velocimetry. Meas Sci Technol 12:1181-1186

Baird DC (1995) Experimentation, 3rd edn. Prentice Hall, Englewood Cliffs

Bardet PM, Peterson PF (2008) Options for scaled experiments for high temperature liquid salt and helium fluid mechanics and convective heat transfer. Nucl Technol 163:330-357

Bindra KS, Chari R, Shukla V, Singh A, Ida S, Oak SM (1999) Twophoton absorption and nonlinear refraction in commercial colour glass filters. J Opt A-Pure Appl Opt 1:73-76

Cho YC (1989) Digital image velocimetry. Appl Optics 28:740-748

Faugeras O (1993) Three-dimensional computer vision: a geometric viewpiont. MIT Press, Cambridge

Gonzales RC, Woods RE (2001) Digital image processing, 2nd edn. Prentice Hall, Englewood Cliffs, pp 270-278

Grant I, Zhao Y, Tan Y, Stewart JN (1991) Three component flow mapping: experiences in stereoscopic PIV and holographic velocimetry. In: Dybbs A, Ghorashi B (eds) Laser anemometry advances and applications, vol 1. ASME, New York, pp 365-371

Grant I, Zhao Y, Smith GH, Stewart JN (1995) Split-screen, singlecamera, laser matrix, stereogrammetry instrument for topographical water wave measurements. Appl Optics 34:3806-3809

Gulec K, Abdou MA, Moir RW, Morley NB, Ying A (2000) Novel liquid blanket configurations and their hydrodynamic analyses for innovative confinement concepts. Fusion Eng Design 59-60: $567-576$

Hecht E (1998) Optics, 3rd edn. Addison-Wesley, Reading

Hoffman PH, Muck KC, Bradshaw P (1985) The effect of concave curvature on turbulent boundary layers. J Fluid Mech 161:371403

Huang HT, Fiedler HE, Wang JJ (1993a) Limitation and improvement of PIV, part I: limitation of conventional techniques due to deformation of image patterns. Exp Fluids 15:168-174

Huang HT, Fiedler HE, Wang JJ (1993b) Limitation and improvement of PIV, part II: particle image distortion, a novel technique. Exp Fluids 15:262-273

Jacob JD, Savaş Ö (1995) Experimental-evidence for intense vortical structures in grid turbulence. $\mathrm{Z}$ Angew Math Phys 46:S699-S716

Keane RD, Adrian RJ, Zhang Y (1995) Super-resolution particle image velocimetry. Meas Sci Technol 6:754-768

Lawson NJ, Wu J (1997) Three-dimensional particle image velocimetry: experimental error analysis of digital angular stereoscopic system. Meas Sci Technol 8:1455-1464

Lin HJ, Perlin M (1998) Improved methods for thin, surface boundary layer investigations. Exp Fluids 25:431-444

Mayer S (2002) A generalized processing technique in digital particle image velocimetry with direct estimation of velocity gradients. Exp Fluids 33:443-457

Moir RW et al (1994) HYLIFE-II: a molten-salt inertial fusion energy power plant design-Final report. Fusion Technol 25:5-25

Oak SM, Bindra KS, Chari R, Rustagi KC (1993) Two-photon absorption in semiconductor-doped glasses. J Opt Soc Am B 10:613-619

Ortega JM (2001) Stability characteristics of counter-rotating vortex pairs in the wakes of triangular-flapped airfoils. $\mathrm{PhD}$. dissertation, Department of Mechanical Engineering, University of California, Berkeley, CA

Pemberten SJ, Abbott RP, Peterson PF (2003) Annular vortex generation for inertial fusion energy beam-line protection. Fusion Sci Technol 43:378-383

Peskin C (1972) Flow patterns around heart valves. Ph.D. thesis, Albert Einstein College of Medicine, Yeshiva University, New-York

Peterson PF (2001) Design methods for thick-liquid protection of inertial fusion chambers. Fusion Technol 39:702-709

Prasad AK (2000) Stereoscopic particle image velocimetry. Exp Fluids 29:103-116

Racca RG, Dewey JM (1988) A method for automated particle tracking in a three-dimensional flow field. Exp Fluids 6:25-32

Sholl MJ, Savaş Ö (1997) A fast Lagrangian PIV method for study of general high gradient flows. In: Proceedings of 35th aerospace sciences meeting/measurement technology, Reno, NV, January 6-9. AIAA Paper No. 97-0493

Shumway RH, Stoffer DS (2006) Time series analysis and its applications, 2nd edn. Springer, New York, pp 23-29

Stoots C, Becker S, Condie K, Durst F, McEligot D (2001) A largescale matched index of refraction flow facility for LDA studies around complex geometries. Exp Fluids 30:391-398

Westerweel J, Nieuwstadt FTM (1991) Performance tests on 3-dimensional velocity measurements with two-camera particle-image velocimeter. In: Dybbs A, Ghorashi B (eds) Laser anemometry advances and applications, vol 1. ASME, New York, pp 349-355

Willert C (1997) Stereoscopic digital particle image velocimetry for application in wind tunnel flows. Meas Sci Technol 8:1465-1479

Wolberg G (1990) Digital image warping. IEEE Comp Soc Press, Los Alamitos, CA

Yu SS et al (2003) An updated point design for heavy ion fusion. Fusion Sci Technol 44:266-273 